\title{
PROPAGATION OF THE ULTRA-SHORT LASER PULSES THROUGH THE HELICAL 1D PHOTONIC CRYSTAL STRUCTURE WITH TWIST DEFECT
}

\author{
Dmitrii V. Antonov ${ }^{1}$, Roman legorov ${ }^{1, a}$ \\ ${ }^{1}$ National Research Tomsk Polytechnic University, 634050 Tomsk, Russia
}

\begin{abstract}
The presence of the photonic band-gap is a featured property of the cholesteric liquid crystals (CLC). It can be practically realized for almost any reasonable wavelengths with very high degree of tunability. We have investigated theoretically the influence of the twist defect of the CLC helical structure onto the bandwidth-limited ultra-short laser pulse propagating inside the photonic band-gap. The changes of both pulse duration and peak power with defect angle were observed together with pulse acceleration and retardation for a case of normal incidence of the light.
\end{abstract}

\section{Introduction}

Photonic structures are of interest in modern optical society due to its ability of highly selective transmission of the light with certain wavelength, polarization and propagation direction [1-3]. One of the simplest and practically available types of 1D photonic crystal is the planar layer of the cholesteric liquid crystals in case when the helix period, refractive indexes and light wavelength values altogether satisfy the conditions of Bragg reflection [2,3]. Thus such layer can reflect almost all the light with circular polarization which handedness is the same as cholesteric helix handedness. The orthogonally polarized light can propagate through the CLC layer without essential losses in case if liquid crystals (LC) are transparent at this wavelength [4,5].

The photonic properties of the CLC are widely used now but mostly with relatively low-power continues wave laser light. The ultra-short laser pulses are existing in the reality that has very small intersection with the world liquid crystalline technologies. Historically it appears because the high power light can destruct the ordering of the mesophase of LC moving it to liquid like state. However, last year's there are some models of LC-based spatial light modulators become available for shaping of the ultra-short pulses [6]. But the application of the CLC for manipulations of the ultra-short laser pulse properties is still not very popular [7]. The experience of the authors said that enough much of pulsed laser solutions are connected with moderate power level [8] and there are a lot of ways to use the CLC-based devices.

At another hand, periodic structures are of special interest for ultra-fast laser community because the strong time compression (stretching) of ultra-short pulses is mostly realized now for the expense of prominent dispersion properties of diffractive gratings $[6,8]$. The selective transmission of the CLC in the Bragg-reflection mode can be interesting from the point of spectral compression (stretching) of light pulses too [8]. Consequently, the application of the CLC layers for an ultra-short pulse management can give good addition to currently used methods.

In this paper we presents the first results of our theoretical investigations about the usage of artificial twist defect of helical structure of CLC $[9,10]$ for simple laser pulse management in the case of normal light incidence onto CLC layer. We simulate the normal propagation of the bandwidth-limited ultra-short laser pulses through the planar CLC layer with twist-defect. We neglect the influence of LC molecular dispersion and absorption onto the pulse parameters because (i) in this work we are looking just for structure induced effects and (ii) it is easy to find the transparent LCs for almost any reasonable wavelengths. The non-linear effects are out of our consideration because most of the popular LC matrices do not show the non-linearity at average light intensity less than $5 \mathrm{~kW} / \mathrm{cm}^{2}$ that corresponds to typical case of unfocused laser beam for low-power devices.

The defects of periodical structures of photonic objects can add the new properties to well-known systems. Additionally, some types of defects allow easy changes of transmission band parameters and, so, it gives us the tunability in potential application of observed effects. Such tunable systems are evidently more attractive for commercial application than permanent one.

\footnotetext{
${ }^{\text {a }}$ Corresponding author: rommel@tpu.ru
}

This is an Open Access article distributed under the terms of the Creative Commons Attribution License .0, which permits unrestricted use, distribution, and reproduction in any medium, provided the original work is properly cited. 


\section{EPJ Web of Conferences}

\section{The simulation procedure}

It is well known, that chiral molecules at certain conditions can form the stratified medium where each molecular layer is rotated relatively previous one for a small angle and therefore make the spatial helix [4,5]. It is evident, that both principal transversal refractive indices of this helical structure are periodically modulated along the helix axis due to the anisotropy of the LC molecules (uniaxial in our case). The period (pitch) of the spiral is usually determined by the concentration of chiral dopant in LC mixture. The center of the Bragg reflection band appears at the light wavelength $\lambda_{C}$, when the average refractive index $\langle n\rangle$ and spiral pitch $P$ satisfy the relation (1). The width $\Delta \lambda$ of the Bragg band-gap could be estimated from (1), where $\Delta n=n_{e}-n_{o}$ is anysotropy of the LC. The overall view of the optical properties of the system is presented at the fig. 1a and fig. $1 \mathrm{~b}$.

$$
\left\{\begin{array}{l}
\lambda_{C}=\langle n\rangle \cdot P \\
\Delta \lambda=\Delta n \cdot P
\end{array}\right.
$$

The light propagation through the uniform planar CLC layer in case of normal light incidence (along the helix axis that coincides with Z-axis of our coordinate system) could be simulated by exact solution of the Maxwell equations as it is shown at [5,9]. This method was proven by numerous comparisons with experimental data [5,9]. The refractive indices of the CLC were chosen as for commercially available E7 mixture $\left(n_{e}=1.72, n_{o}=1.51\right)$. The cholesteric pitch was chosen $P=392 \mathrm{~nm}$ that corresponds to $\lambda_{C}=633 \mathrm{~nm}$.

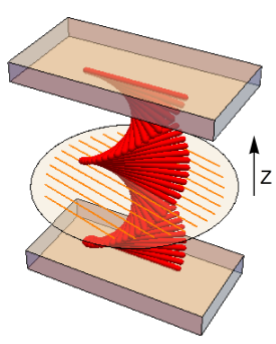

a

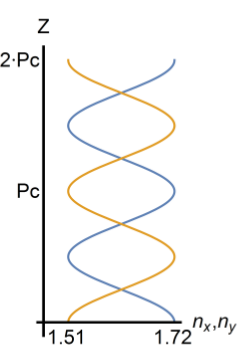

b

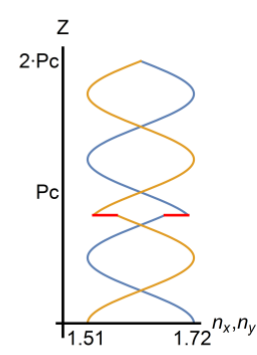

C

Figure 1. The molecular alignment of the uniform planar cholesteric layer between glass substrates (a). The modulation of the refractive indexes $n_{x}$ (blue) and $n_{y}$ (yellow) along the Z-axis due to the rotation of the LC molecules in the case of uniform helix (b) and the same (c) for a case with $90^{\circ}$ twist defect (the place of a defect is shown by red).

In the defect-free case the transmission spectra of the CLC layer shows the very different transmission for the right-handed (RHCP) and left-handed circularly polarized (LHCP) light inside the Bragg band-gap. The typical structure-induced transmission spectra are shown at the inset of fig. 2. The transmission for both components inside the band-gap has relatively small changes in range $\lambda_{C} \pm \Delta \lambda / 2$. The thicker CLC layer with higher $\Delta n$ corresponds to the sharper edges of the band-gap. For a case of the E7 mixture we see the band-gap width more than $80 \mathrm{~nm}$. So, such layer is a simple one-dimensional photonic crystal with selective propagation of orthogonal circularly polarized light.

The 1D-photonic crystal with defect was assumed as two consequent similar thickness layers (the thickness of each semi-layer is $d=10 \mathrm{mkm}$ ) of the planar oriented CLC with the second semi-layer rotated for certain angle $\Delta \varphi$ around cholesteric helix axis (Z-axis). This leads to certain jump of the refractive indices modulation as it schematically shown at fig. 1c. The transmission of such composite layer can be easily simulated by multiplication of $4 \times 4$ transfer matrices that corresponds to each layer as shown [9]. The introduced non-uniformity of the molecular alignment leads to appearance of the additional defect-induced Eigen modes inside the CLC layer and corresponding modification of a transmission spectrum (see fig. 2). The changes of the twist-defect angle $\Delta \varphi$ from zero and up to $90^{\circ}$ leads to appearance of additional narrow reflection bands (for a light component that is usually well transmitted) with different depth that appear at right border of photonic band-gap and slide to its left side with $\Delta \varphi$. The $\Delta \varphi=90^{\circ}$ corresponds to additional reflection band at the center of the CLC band-gap which achieves nearly zero transmission.

Switching to the light pulses propagation, we have to take in account that enough strong phase retardation of the different spectral components present together with changes of the transmission value. Thus in common case, every partial monochromatic wave has own unique changes of the amplitude and phase after the propagation through the CLC layer. This introduces certain chirp to the pulse but it is not valuable in the case of relatively narrow pulse spectrum together with small thickness of the CLC layer.

For the simplicity we assume that pulse has normalized Gaussian spectrum and therefore the same typical Gaussian shape in time-domain. The central wavelength of the incident laser pulse was chosen as $633 \mathrm{~nm}$ and pulse bandwidth was $\sim 6 \mathrm{~nm}$ that corresponds to $227 \mathrm{fs}$ of bandwidth-limited duration. These numbers are not principal because the CLC can be prepared for another wavelengths in visible or NIR bands too. The structure-induced effects will appear the same for other wavelengths if we have similar ratios between $P, \lambda_{C}, \Delta \lambda, d$ and the same defect position relatively overall layer thickness. The polarization of the spectral components of the incident pulse was defined as RHCP that allows us to refine the results respectively to the polarization induced complexity without losses of the main information. This is enough good assumption because the pulse bandwidth is relatively narrow and the light ellipticity at the Gaussian wings of the real pulses is not very different from $100 \%$.

The components of the pulse spectrum (that was simulated with 1000 points) were multiplied by the corresponding complex transmission coefficients of the CLC layer. The output spectrum (see fig.3a) was transformed to the time-domain view using Fast- 
Fourier-Transform. The full spectral window was chosen so that step in time-domain was less than $5 \mathrm{fs}$. It gives enough good level of detalization of pulse shape together with enough wide time-domain window.

The output light is the same RHCP as an incident one because the Eigen-modes of the CLC layer have polarization which is very close to circular.

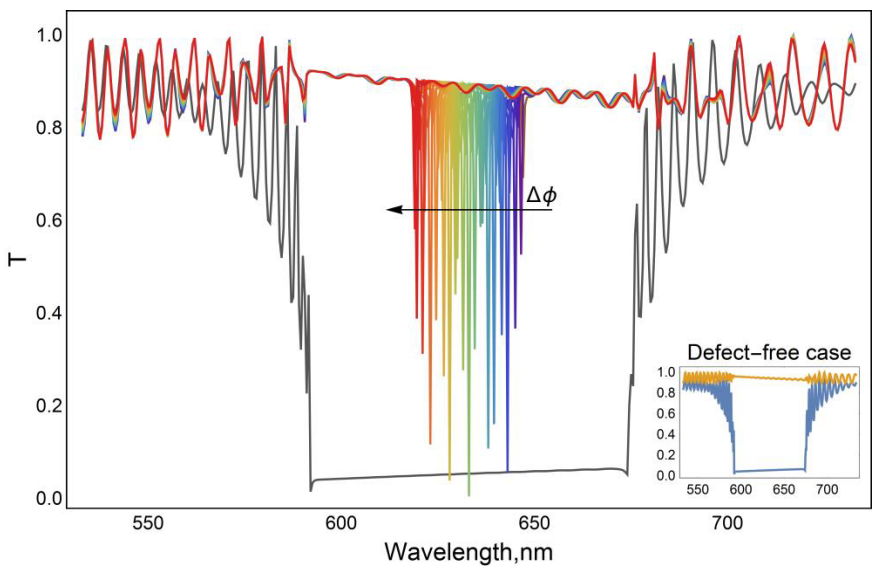

Figure 2. The intensity transmission spectra of the CLC layer with different twist defects $\Delta \varphi \in\left[70^{\circ}-110^{\circ}\right]$. The transmission of the mostly reflected LHCP component is shown by black, the spectra of the transmitted RHCP component is shown by rainbow curves with $\Delta \varphi$ growing from blue to red, along the arrow. The inset shows the transmission spectra for defect-free case $(\Delta \varphi=0)$ : RHCP is yellow curve and LHCP is blue.

\section{Results and discussion}

Some examples of spectra of the output pulses for different twist defect angles are shown at fig. 3a. The modification of the pulse spectrum by the CLC transmission band profile is the main mechanism of the pulse transformation here. The defect-induced reflection peak moves with $\Delta \varphi$ right to left and introduces first the changes at right wing and further at the central part of pulse spectrum. The left wing has a changes when $\Delta \varphi>90^{\circ}$.

The output pulses demonstrate changes of durations, amplitudes and different retardation with changes of the defect angle. The fig. $3 \mathrm{~b}$ shows the pulse duration changes. As one can see, the pulse becomes for $1 \%$ longer than for defect-free case and after this its duration decreases for approx. $4 \%$ near $\Delta \varphi=90^{\circ}$ with following growth. It could be easy explained if we look onto the spestral bandwidth changes: it is decreased first when defect-related reflection band touch the pulse spectrum wings and it grows when the defect band go to the center of the pulse spectrum and decrease its peak value conserving the full width at half maximum. Our input pulse is transform-limited and the thickness of a CLC layer is very small, so the output pulse does not collect essential chirp and its duration is inversely proportional to the spectral bandwidth. This is so-called spectral stretching and compression of a pulse.
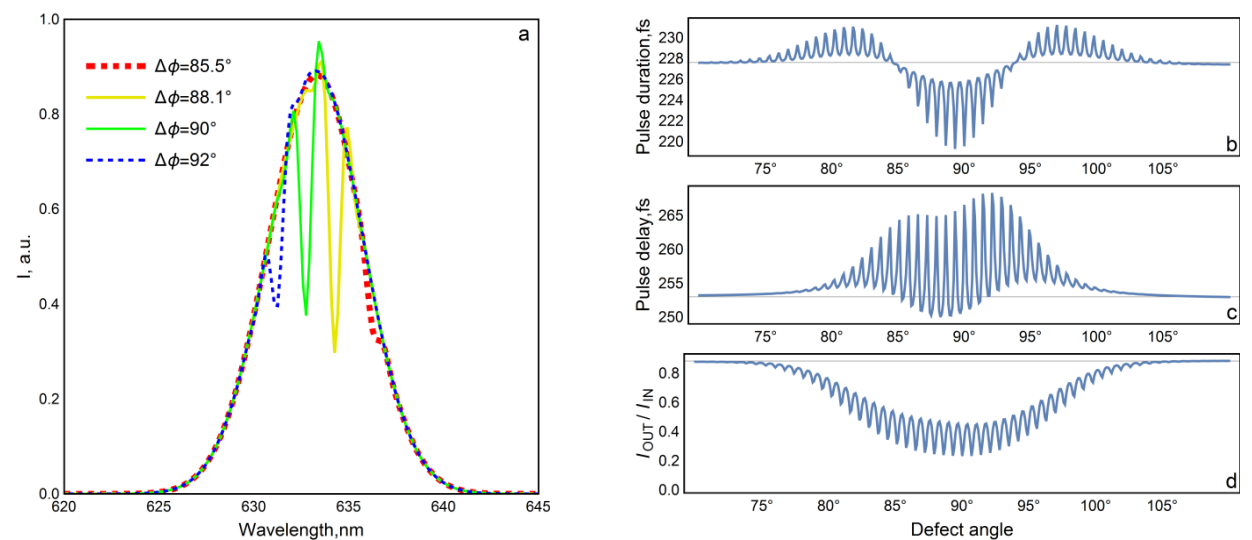

Figure 3. The examples of the output pulse spectrum for the different defect angles (a). The influence of the twist defect of the CLC onto the duration of the output pulse (b). Changes of the pulse delay with defect angle (c), changes of the pulse peak power with defect angle (d). The horizontal grid-lines show the values corresponding to the defect-free case. 


\section{EPJ Web of Conferences}

The pulse amplitude is shown at fig. 3c. The behavior of this dependence could be qualitatively explained very easy too. The defect-induced reflection band goes with $\Delta \varphi$ from the right wing of the spectrum to its center and further to left wing. Due to the gaussian shape of the spectrum we have the maximal decrease of the light power when the defect reflection band is near the peak of the pulse spectrum.

The most interesting behavior has the defect-induced pulse retardation. As one can see from fig. $3 \mathrm{~b}$, pulse first become retarded for up to $4 \%$ relatively defect-free case but before $\Delta \varphi=90^{\circ}$ it demonstrates acceleration when delay is $2 \%$ less than for defectfree case. This effect is connected with essential power re-distribution inside the pulse when center of weight of a pulse has shifted from its initial place showing the effective growth of the pulse group velocity. The tunable pulse retardation with different sign of an effect can be utilized for different applications.

It is worth noting that all these effects which are visually small by absolute value, has appeared at just $20 \mathrm{mkm}$ overall thickness of the CLC layer. This is equivalent of just 30-40 wavelengths and even taking in account the thickness of a glass substrates of the LC-cell this don't need more than some millimeters of a space. Thus the devices that utilize the presented approach could be a very compact and enough flexible solution for fine manipulation of the pulse parameters.

The fine structure of presented at fig. 3 dependencies has appeared as result of interference nature of photonic band-gap phenomena. The interesting feature of this high frequency modulation is total anticorrelation of oscillations of pulse retardation and pulse amplitude dependences for all range of the $\Delta \varphi$ changes. However, the pulse duration is anticorrelated with pulse amplitude just at ranges where pulse is stretched relatively defect-free case. And these oscillations are correlated at the range where pulse is compressed by defect. It means that we can't have together the minimal duration and maximal peak power of a pulse but we can have shortest pulses together with shortest time of its propagation inside the CLC layer.

The comparison of different curves shows that strong pulse compression could be achieved only together with high losses of pulse energy - shortest output pulse corresponds to decrease of the peak power for four times. However, this is common feature of all popular techniques of pulse compression or stretching. For example, the best grating compressor (that is built on very expensive ruled gratings) introduce at least $30-40 \%$ of power losses.

\section{Conclusions}

The obtained results show that introduction of the defects to the photonic structure of CLC can lead to evident changes of its optical properties regarding transmission of the ultra-short laser pulses. The offered type of defects (twist defects) allows very easy manipulation of the parameters of transmitted laser pulse just by relative rotation of two CLC sub-layers around the axis which is perpendicular to the cell substrates.

Both pulse duration and peak power as well as the pulse retardation are sensitive for defect angle changes in range 75-105 degrees. The bandwidth-limited pulse could be stretched for $1 \%$ as well as compressed for $4 \%$ at presented parameters of the CLC layer. The pulse retardation induced by defect could be positive as well as negative depending on the defect angle.

The presented results can be used as basement for creation of more complicated photonic structures with more than one defect that gives higher level of flexibility and tunability together with higher range of changes of pulse parameters.

This work supported by the Grant of TPU VIU_ENIN_94_2014.

\section{References}

1. E. Yablonovitch, T.J. Gmitter, K.M. Leung, E; Gmitter, TJ; Leung, Phys. Rev. Lett. 67 (17), 2295-2298, (1991).

2. Kolle, Mathias, "Photonic Structures Inspired by Nature" (1st ed., Springer Theses, 2011)

3. John D Joannopoulos, Johnson SG, Winn JN \& Meade RD, "Photonic Crystals: Molding the Flow of Light" (2nd ed., Princeton NJ: Princeton University Press, 2008).

4. P. G. de Gennes, J. Prost, "The Physics of Liquid Crystals", (Clarendon Press, 1995).

5. Ingo Dierking, Symmetry, 6, 444-472, (2014).

6. Svelto Orazio, "Principles of Lasers" (5-th ed., Springer US, 2010).

7. L. Song, S. Fu, Y. Liu, J. Zhou, V. G. Chigrinov, and I. Khoo, Opt. Lett., 38, (23), 5040-5042, (2013).

8. Govind P. Agrawal,"Nonlinear Fiber Optics" (4-th ed., Rochester, New York, 2006).

9. Egorov, R. I., Kiselev, A. D., App. Phys. B, 101, (1-2), 231-234, (2010).

10. Kopp V.I., Genack A.Z., Phys. Rev. Lett., 89, (3), 339011-339014, (2002). 